

\title{
Testing the additive independence assumption in the QALY model
}

\author{
Anne Spencer* \\ Queen Mary, University of London
}

November 2000

\begin{abstract}
Quality-Adjusted Life Years (QALYs) estimate the utility derived from health states by taking account of life expectancy and quality of life. In applying QALYs to situations where health varies over time, it is usual to assume that we can add the utilities from constituent health states. This assumption of additive independence has been challenged by research suggesting that people's preferences for health states are affected by the sequence in which health states occur. This paper investigates two tests of additive independence as well as a test of the consistency of preferences over time. The main test of additive independence is based on a new method using stylised health profiles of deteriorating, improving or temporarily-improving health. The advantage of this new method is that the test relies only on a comparison of health states occurring in the same time period and therefore controls for the effects of time preference. The other test of additive independence determines whether people strictly prefer one type of health profile over another and collects qualitative data on the issues considered by people in these choices. In the main test of additive independence, only one of the two cases considered detected a statistical difference. In the other test of additive independence, the sample was split almost equally between those strictly preferring one or other of the choices. The qualitative data revealed contrasting viewpoints about the benefits derived from such choices which led to this split. The paper also found that preferences were consistent over time. The tests of additive independence were, therefore, unable to conclusively reject additive independence.
\end{abstract}

JEL classification: $\mathrm{I} 31$

Key words: utility measurement, additive independence, health profiles

* Department of Economics, Queen Mary, University of London, Mile End Road, London, E1 4NS.Tel: 020-7882-5532; Fax: 020-8983-3580; e-mail address: a.e.spencer@qmw.ac.uk. 


\section{Introduction}

Applied work that assesses the cost effectiveness of different treatments faces the challenge of how best to measure the benefit when health varies over time. Quality adjusted life-years (QALYs) have been developed by health economists to take account of both quality of life and life expectancy. Pliskin, Shepard \& Weinstein (1980) outline the assumptions required for QALYs to be a valid measure of preferences under expected utility (EU) theory (a critique of these assumptions is given by Loomes \& McKenzie, 1989). When health varies over time, Bleichrodt (1995) has shown that for QALYs to be still a valid measure under EU it is necessary to add the assumption that we can simply add the preference scores or values from constituent health states. The addition of utility values is the conventional method to estimate preferences for treatment profiles that offer variations in health. As a challenge to this assumption of additive independence, research has suggested that people's preferences are also affected by the sequence in which health occurs (Loewenstein \& Prelec, 1993). MacKeigan, O'Brien \& Oh (1999) found that the additive independence assumption held for profiles with gradual deterioration in health. MacKeigan et al. (1999) recommend further work to test whether their result is replicated in a broader category of health profiles. The main aim of this paper, therefore, is to develop a test of this assumption for a broad category of health profiles offering deteriorating, improving or temporarily-improving heath. In addition, it offers a simple technique to control for time preference in tests of additive independence.

Few papers consider preference elicitation in the more realistic situation where health varies over time. The debate about estimating profiles is linked with research that looks at holistic valuations - for instance, the Healthy Years Equivalent method (HYE). This interesting development has often been overshadowed by the debate on the equivalence between the HYE and other measures (Bleichrodt, 1995). The ability to estimate profiles simply from constituent states reduces the cost of estimation. For instance, in the EuroQol classification system, valuing all possible combinations of profiles over a ten year period would require the estimation of $243^{10}$ profiles. 
However, if these simpler approaches are to be widely accepted, it is important to offer empirical results to support additive independence.

The new test of additive independence reported here adopts a novel approach for controlling for the possible effects of time preference. Part of the problem with devising tests of additive independence is the difficulty of distinguishing between preferences for the sequence of health and for the timing of health. The new test relies simply on looking at differences between preferences for health profiles. The differences between profiles then determine the extent to which the same constituent health states are viewed differently depending upon the profiles in which they are embedded. We investigate the extent to which this new test replicates the results from other studies. The paper considers another test of additive independence and a test of the consistency of preferences over time. Both these tests collect qualitative data alongside quantitative data.

The secondary aim of the paper is to see the extent to which the qualitative data reveal trends in the quantitative data. Johnston, Brown, Gerard, O’Hanlon \& Morton (1998) have recommended but not used such an approach after conducting a quantitative analysis of utility values for temporary and chronic health states of breast screening outcomes. However, there have been relatively few joint qualitative and quantitative studies of value elicitation procedures (we are aware of four joint papers in this area: Dolan \& Cookson 2000; Dolan, Cookson \& Ferguson 1999; Beattie, Covey, Dolan, Hopkins, Jones-Lee, Loomes et al. 1998; Robinson, Dolan \& Williams 1997). This paper will investigate the extent to which the qualitative data can add to our understanding of the quantitative data.

\section{Overview of existing tests of additive independence}

We consider the studies that incorporate a test of additive independence, concentrating on the methods they used and the extent to which sequencing effects are found (see Spencer, 1999). 


\section{Estimation of profiles from constituent states}

Dolan \& Gudex (1995) compared the values implied for temporary states with the values for chronic states using the Time Trade-Off (TTO) method. The TTO method compared two health profiles. For instance, the respondent was required to imagine suffering a particular health state for the entire duration of the profile. They were then asked to compare this profile against an alternative health profile which included a combination of two states, usually normal health and death. The time in normal health was varied until they were indifferent between the two profiles (Torrance, 1986). The temporary states considered by Dolan \& Gudex (1995) lasted for one month or a year, and were embedded in an otherwise healthy ten year profile, whereas the chronic states continued for the entire 10 years. They found that the values for temporary states were significantly lower than the values for chronic states. One reason for this is that although responses were adjusted upwards to reflect a preference for shorter periods in poorer health, these adjustments were insufficient to give higher implied values for temporary states. Another reason may be the failure of additive independence. This appears a strong possibility, since the authors reported that $35 \%$ of their sample showed a negative discount rate, suggesting that the sequence, as well as timing of states, may affect preferences. For instance, people may prefer to undergo poor health now and look forward to good health in the future.

Richardson co-authored two papers (1989 and 1996) in this area and used TTO, Standard Gamble (SG) and Visual Analogue Scale (VAS) methods. In the SG method, the choice was between remaining in a health state or undergoing a risky treatment. This treatment had probability $\mathrm{p}$ of succeeding, resulting in a better health state (say normal health), or (1-p) of failing, resulting in a worse health state (say death). Probability $\mathrm{p}$ was varied until the respondent was indifferent between the two alternatives. In the VAS method, the respondent simply placed the health states on a scale from 100 to 0 to represent their degree of severity (where normal health is 100 and death is 0). Richardson, Hall \& Salkeld (1996) asked respondents to rank and value four health states $^{1}$ : a practice state and three main states which were mild, moderate and severe ${ }^{2}$. They 
were then asked to value a profile which lasted for 16 years and represented a gradual deterioration: 5 years in a mild state, 10 years in a moderate state and 1 year in a severe state. The severe state was placed at the end. They then combined the constituent states to estimate the profile using a discount rate of 3\% or 9\% (Richardson, Hall \& Salkeld 1989 used a discount rate of $0 \%, 5 \%$ and $10 \%$ ). They found that using either the VAS, TTO and SG methods the constituent states overestimated the profiles by between $31 \%$ and $57 \%$ and that it was not possible to find a plausible discount rate that would suggest equivalence.

Mackeigan et al. (1999) used the TTO method to value profiles and their constituent states, and compared the extent to which they differed. They asked 101 diabetes patients for their preferences towards different treatments lasting 30 years, with 4 possible deteriorating treatment profiles. The profile was not statistically different from the constituent states. This result contrasts with the work that has gone before, and the authors conclude that this may be because the profiles were too similar. They recommend that research is repeated in profiles that are more distinct and where sequencing effects are likely to be larger.

\section{Econometric analysis to discern the correct utility function}

Lipscomb (1989) used regression analysis to predict the extent to which different components affected the valuation. Part of this modelling allowed him to look at interaction terms between the different health states. The regression showed significant interaction terms which suggested that a simple additive model was inappropriate. He confirmed Richardson's result that, given three different discount rates $(0 \%, 5 \%$ and $10 \%)$, profiles for this representative respondent could not easily be estimated by a weighted average of constituent health states.

Kuppermann, Shiboski, Feeny, Elkin, \& Washington (1997) compared profiles against constituent health states for patients at a maternity clinic who were considering prenatal diagnosis. They used two additive models: one model assumed that it was appropriate to 
multiply quality and survival duration (termed duration weighted additive model) and the other was a more general model derived from statistically inferred weights (termed a multiple regression model). Neither model considered interaction between health states in the same profile (see Kuppermann et al., 1997 table 4) ${ }^{3}$. A total of 121 women took part. Their article concentrates on the VAS and SG values of 43 women, who were asked to consider constituent states over 4 time periods and who selected their own worst state. Throughout, they used a zero discount rate, but found that the result was unaffected by changing this to a $5 \%$ discount rate. The general additive model was found to predict the profiles better than the additive model ${ }^{4}$ but as Holmes (1998) points out, such weights have no underlying conceptual basis in terms of the conventional QALY approach.

\section{Experimental tests of the additive independence axiom}

Krabbe \& Bonsel (1998) tested the impact of good health appearing early or later in the treatment. They asked 104 students to value 13 EuroQol states (Kind, Dolan, Gudex \& Williams, 1998) using two types of TTO questions, both based on a ten year period. The standard-sequence TTO question offered a treatment which lead to the 'best imaginable state' first and 'worst imaginable state' last and the reverse-sequence TTO offered the 'worst imaginable state' first and the 'best imaginable state' last. When there is zero discounting each health state should be valued equally using the standard or reverse sequence TTO question. When differences arise these could occur because of discounting or sequencing effects. They attempted to control for discounting by imposing a discount rate that led to the smallest difference between the standard-sequence and reverse-sequence TTO questions at group level (they used a group voting system to elicit the value). The residual difference should, therefore, give an estimate of the sequencing effects under this restriction. They found evidence of a sequencing effect, with 6 of the 13 profiles reverse-sequence TTO questions led to lower values, which is in keeping with the finding that respondents place a higher value on good health at the end of the profile. 


\section{Summary}

Most of the tests of additive independence have relied upon checking the extent to which it is possible to estimate profiles from constituent states. Such tests rely upon imposing a particular discount function (usually assumed to be exponential) and require the estimation of the implied discount rate. The drawback of these papers is that the implied discount rate can be affected by sequencing and discounting effects (Gafni, 1995). A further problem is that experimental test that rely on the TTO method will find that the TTO values themselves are also affected by the interplay of timing and sequencing issues. This suggests that elicitation methods which do not rely on time, such as the SG or VAS methods, are more appropriate for tests of additive independence. This paper tries a new approach to test for additive independence that overcomes some of the problems experienced in these papers.

Trends are beginning to emerge from these empirical papers. Dolan \& Gudex (1995) found that constituent states valued as chronic states, overestimated temporarily-deteriorating profiles and that respondents derived less benefit from a temporary deterioration in health. They suspected that although respondents adjusted upwards to reflect a preference for shorter periods in illhealth, these adjustments were insufficient to give higher implied values for the temporary states embedded in an otherwise healthy profile. In addition, some of the empirical findings support psychological predictions. Psychologists have predicted that respondents would pay more attention to the final health state (Kahneman, Fredrickson, Schreibner \& Redelmeier 1993; Varey \& Kahneman 1992; Ross \& Simonson 1991). Richardson et al. (1996) found that constituent states overestimate deteriorating health profiles and is in keeping with the notion that respondents gave more weight to the end health state reached. Mackeigan et al. (1999), on the other hand, found no evidence of this for profiles offering a gradual deterioration. This could be explained by the gradual adaptation to the new health states and reference point effects (Ross \& Simonson, 1991). The findings of Kuppermann, et al. (1997) are harder to link to these patterns since they considered deteriorating and improving health states within each profile. However, they 
conclude that the profiles could not be accurately estimated using the additive QALY model, but could be estimated by more general regression models. Another prediction of psychologists is that respondents may desire to overcome ill-health and look forward to good health (dread and savouring, Loewenstein \& Prelec, 1993), but so far health studies have not looked at this issue. This paper adds to the empirical research by investigating the extent to which a broad set of profiles, considered within the same questionnaire, replicates the findings of these studies.

\section{Tests of the QALY model when health varies}

This paper contributes to this research by outlining two tests of additive independence and one for the dynamic consistency of preferences. The main test of additive independence compares differences in profiles, and bypasses the problems of the changing attitudes towards time preference encountered in tests that rely on imposing a particular discount function or fail to fully discriminate between discounting and sequencing effects.

\section{A new test of additive independence based on a comparison of differences}

This test controls for a respondent's attitude towards risk as well as time preference whilst manipulating the sequence of states. In addition, it is able to test a wide variety of states which may lead to failures of additive independence such as adaptation to temporary health states and a desire to overcome ill-health and look forward to good health.

To test the additive independence consider three time periods, $t+1, t+2$ and $t+3$, and three health states $\mathrm{N}, \mathrm{Y}, \mathrm{Z}$ denoted by shading in the figures that follow. In the example given in figure 1 respondents consider a ten year period after which they will die. For example, in profile (i) a respondent considers three years in $\mathrm{Z}$, followed by three years in health state $\mathrm{N}$ and four years in health state $\mathrm{N}$, which we denote by $\mathrm{ZNN}$ below. In profile (ii) a respondent considers three years in Z, followed by three years in $\mathrm{Y}$ and four years in Z, which we denote by ZYZ below 
(similarly for the other profiles):

Figure 1 Four health profiles

When additive independence applies the strength of preference for one state over another, in time $\mathrm{t}+2$ say, is independent of the profiles in which the two states are embedded. The additive independence function can be written as equation 1 (see von Winterfeldt \& Edwards, 1986):

$$
\mathrm{U}\left(\mathrm{Q}_{\mathrm{t}+1}, \mathrm{Q}_{\mathrm{t}+2}, \mathrm{Q}_{\mathrm{t}+3}\right)=\mathrm{w}_{(\mathrm{t}+1)} \mathrm{U}\left(\mathrm{Q}_{\mathrm{t}+1}\right)+\mathrm{w}_{(\mathrm{t}+2)} \mathrm{U}\left(\mathrm{Q}_{\mathrm{t}+2}\right)+\mathrm{w}_{(\mathrm{t}+3)} \mathrm{U}\left(\mathrm{Q}_{\mathrm{t}+3}\right)
$$

where $\mathrm{w}_{(\mathrm{t}+\mathrm{i})}$ is the discount function at time $\mathrm{t}+\mathrm{i}, \mathrm{Q}$ is the health state and $\mathrm{U}($.$) is the utility$ function

This implies that the difference between profiles (i) and (ii) should be the same as the difference between profiles (iii) and (iv) (see appendix 1). The advantage of this approach is that the test relies only on a comparison of health states occurring within the same time period, therefore, it controls for the effect of time preference and different attitudes towards the risk of premature death. At the same time as this experiment was being conducted, a similar approach to control for the influence of time preference was reported by Treadwell (1998). The main difference between his approach and ours is that he tested whether changing the health state in the same period changed respondents' choices between alternative profiles. His experiment, therefore, was designed to test more general models of preference independence.

A minimum of two states is required for this test. Two states have the advantage of simplicity, but would preclude the possibility of considering the effects of more complicated patterns, for instance declining or improving states. 


\section{A further test of additive independence based on a comparison of two risky treatments}

Bleichrodt (1995) suggests a test for additive independence that is similar to that proposed by Pliskin et al. (1980) to test for additive independence between health states and survival duration in the first-stage estimation. Consider the two treatments shown in figure 2. The respondent is asked to imagine that they became ill and are offered a choice between two treatments. No treatment would result in them remaining in health state ZZZ. In treatment $C$, they have a $50 \%$ probability of it succeeding and returning to $\mathrm{ZNN}$ over the next ten years and a $50 \%$ probability of it failing and resulting in NZZ. In treatment $\mathrm{D}$, they have a $50 \%$ probability of it succeeding and returning to full health for the next ten years and a $50 \%$ probability of it failing and then remaining in health state $\mathrm{Z}$ for the next ten years. The respondent is asked which they prefer or, if they do not mind, which treatment they receive, and asked to give their reasons. If additive independence holds, then the respondent is indifferent between the treatments since, in effect, the two treatments offer the same expected utility i.e. $0.5 \mathrm{U}\left(\mathrm{Z}_{\mathrm{t}+1}, \mathrm{~N}_{\mathrm{t}+2}, \mathrm{~N}_{\mathrm{t}+3}\right)+0.5 \mathrm{U}\left(\mathrm{N}_{\mathrm{t}+1}, \mathrm{Z}_{\mathrm{t}+2}\right.$, $\left.\mathrm{Z}_{\mathrm{t}+3}\right)=0.5 \mathrm{U}\left(\mathrm{N}_{\mathrm{t}+1}, \mathrm{~N}_{\mathrm{t}+2}, \mathrm{~N}_{\mathrm{t}+3}\right)+0.5 \mathrm{U}\left(\mathrm{Z}_{\mathrm{t}+1}, \mathrm{Z}_{\mathrm{t}+2}, \mathrm{Z}_{\mathrm{t}+3}\right)$. Bleichrodt (1995) anticipated that more intermittent health states, such as ZNN or NZZ will be preferred to chronic states, such as NNN or ZZZ, but he did collect data on these questions.

Figure 2 Chronic and intermittent health states

In principle, it is possible to manipulate the sequence of states in this test whilst controlling for a respondent's attitude towards risk of death and time preference. The drawback of this approach is that it requires a more complex SG question than usual, which involves two risky treatments. A respondent is likely to be able to express their preferences between the treatments but they may find it difficult to adjust the probability to give an indication of their strength of preference. 


\section{A test of dynamic consistency}

This test is devised to look at the consistency of preferences over time and consider the effect of changing the point at which uncertainty is introduced into the question. In the SG question in figure 3 (termed SG3 in the main questionnaire), in treatment $\mathrm{H}$ the respondent will experience health states YYZ for certain, resulting in a declining profile. In treatment $G$, they experience Y for certain in periods one and two and are offered an uncertain treatment at the beginning of period three. The treatment offers probability $\mathrm{p}$ that it will result in $\mathrm{N}$, and probability $(1-\mathrm{p})$ that it will result in D. The respondent sets $\mathrm{p}$ so that they do not mind which treatment they receive.

Figure 3 Postponing treatment in question SG3

Figure 4 The implied SG3 question

If the utility value can simply be added across the states, the SG question in figure 3 is equivalent to the SG question in figure 4. For instance, question SG3 will yield an expected utility of $U\left(Y_{t+1}\right.$, $\left.Y_{t+2}, Y_{t+3}\right)=U\left(Y_{t+1}, Y_{t+2}\right)+p U\left(N_{t+3}\right)+(1-p) U\left(D_{t+3}\right)$ whilst the implied SG3 question yields an expected utility of $U\left(Y_{t+1}, Y_{t+2}, Y_{t+3}\right)=p^{\prime} U\left(Y_{t+1}, Y_{t+2}, N_{t+3}\right)+$

(1-p') $U\left(Y_{t+1}, Y_{t+2}, D_{t+3}\right)$. These are equivalent when $p=p^{\prime}$. Notice, however, that this question may result in changes in a respondent's attitudes towards the risk of premature death, since postponing treatment draws attention to the last four years of their life, even though they are asked to set that level of probability for this at the beginning of the ten year period. This test, therefore, cannot control for the effects of changing attitudes towards risk that may arise from the reframing of the question.

\section{Overview of the questionnaire}

The study uses the EuroQol classification system which describe states of health along five dimensions: mobility, self-care, usual activities, pain and anxiety. Each dimension has three 
levels of severity: no problems, some problems and more-severe problems, denoted by 1,2 and 3 respectively and colour-coded black, blue and red in our study. Each health state is colourcoded and we refer to these as follows: 11111 as N, 12221 as W, 21222 as Y, 22232 as Z and death as D. The state 12221 allows respondents to become familiar with the methods, but the utility values are not used in the study.

The respondents are asked to imagine that each profile lasts for ten years without change, to be followed immediately by death. They are then asked to consider treatments leading to changes of health over the next ten years. It is explained to respondents that each profile consists of three periods: the first three years, the second three years and the last four years. Health states are constant in any one period. The respondents are asked to consider at most three different health states in the ten year profile (after which they would die).

The cards are passed to respondents one at a time and they are asked to rank them. This is followed by 11 SG questions (including a consistency check, question 11) and three adapted SG questions (SG1 to SG3).

Table 1 An overview of the questionnaire

Table 1 summarises the questions: column 1 shows the question number and column 2 the health profiles used in questions 1-10 and the particular research questions that each of the later questions were trying to address. The first three questions are conventional SG questions. Question 1 allows respondents to become familiar with SG questions and questions 2 and 3 enable an estimation of profiles from constituent states.

Figure 5 The format of questions 1 to 10

Questions 4 to 10 investigate respondents' reactions to different profiles, including their reactions 
to: dread/savouring (questions 4 and 5), the speed of recovery/deterioration (questions 7 and 8) and a temporary improvement (question 9). These labels are not unique and the profiles could be regrouped. For instance, question 9 could be an example of deterioration of health in the final period, or a focus on the final health state. The profiles have initially been grouped by changes in key features that seem to distinguish them from other profiles. Qualitative data are collected alongside the quantitative data in an attempt to understand more fully respondents' interpretations of the profiles. Questions 6 and 10 are used to predict the responses to question SG3 described more fully below. Question 11 provides a consistency check for the responses and compares the profile YYY against a risky treatment involving ZNN and death. If responses are consistent, the implied utility value for $\mathrm{Y}$ from question 11 is the same as the implied utility value for $\mathrm{Y}$ from question 2. The respondents are asked to compare each of the first ten profiles against normal health and death and are shown in figure 5. They are asked to state the chance of success and failure where they consider the alternatives to be most finely balanced and they do not mind which treatment they receive. To help them with this they are given a sheet of paper listing the chances of success/failure against which they mark their response (based on Jones-Lee, Loomes \& Philips, 1995).

The three adapted SG questions are based on those presented. The first SG question, SG1, asks respondents to consider whether they prefer a treatment that offers more intermittent states, or one that offers chronic states, or if they do not mind which treatment they receive as shown in figure 2 .

The other two adapted SG questions, SG2 and SG3, investigate postponing the point at which uncertainty is introduced. SG2 is a practice question and differs only in that the profile under consideration improves over time. SG3 investigates the effect of postponing the risky treatment until the beginning of the seventh year in a profile that is declining over time as shown in figure 3. Respondents are asked to decide at the beginning of the ten year period the level of success and failure where the two alternatives that are most finely balanced and they do not mind which treatment they receive. The response to SG3 can be estimated from the responses to the profiles 
which make up this question. For example, SG3 can equivalently be expressed as figure 4. The profiles in figure 4 are already estimated by questions 6,4 and 10 respectively, and can be used to calculate an implied probability for SG3.

\section{Data}

Members of the general public were invited to take part in a 60-minute interview in the Department of Economics at York University for a payment of $£ 10^{5}$. In the past studies have elicited the views of patients, members of the general public or policy makers (Drummond et al., 1997). Members of the general public were chosen since the EuroQol measure has already been extensively used to elicit the preferences of these respondents (Kind et al., 1998). For more information on the sample selection issues for qualitative studies see Curtis, Gesler, Smith \& Washburn, 2000. All interviews were tape-recorded. In total, 29 respondents were interviewed, 15 males and 14 females.

Table 2 The sample

In the qualitative data the respondent was asked to think aloud during the questions and to declare the types of information that they were heeding or concentrating using a verbal protocol analysis approach developed by Ericsson \& Simons (1980). In addition, respondents were asked to express the issues that they had been thinking about at the end of questions SG1-SG3. The study made use of clarification probes in one of two ways (Patton, 1990). The interviewer verbalised a respondent's non-verbal cues, such as eye or hand movements which were used to emphasise a point. In addition, an attempt was made to record the health states that were being considered by a respondent at each stage of the question. The comments to questions SG2-SG3 were recorded in the response sheet and were transcribed for all 29 respondents. 


\section{Analysis of the qualitative data}

Given that the use of qualitative data is uncommon in value elicitation more details are given here about the methods used to analyse the qualitative data. The qualitative analysis was based on Glaser \& Strauss's (1967) grounded theory. Their approach encourages a reliance on the data to form new theories and concepts and suggests an inductive approach whereby the researcher infers general laws from particular instances. In addition, it encourages the researcher to actively question the interpretation of the data and to form new theories. This is made clearer in the writings of Charmaz (1990) who emphasised the need to formally acknowledge the perspective from which the analysis is being conducted. The analysis involves summarising the excerpts using the respondent's own terminology and key phrases. These are categorised and given a specific code. This is later followed by grouping specific codes to form general categories which characterise particular themes. An advantage of this approach is that it helps to widen the categories considered by the researcher and retains minority points of view (taxonomy development). It also gives a record of the steps taken by the researcher to reach their conclusions by summarising the specific codes which are combined to form general codes (development of a conceptual framework). Finally, cases, which appear to contradict the general rules, can be identified. In grounded theory there is an effort to seek cases which would contradict the theory, termed negative cases (Kidder, 1981). The transcribed interviewer scripts were analysed using the software package HyperResearch $\subset$ that aids the data management and analysis of non-numerical data. Two potential pitfalls were identified in the efforts to identify themes. The researcher was careful not to read too much into the specific codes. Some of the respondents simply repeated the instructions of the question but this could not be used as evidence that they were focusing upon a particular health state. In some cases, it may be too simplistic a statement to say that respondents were concerned about one issue. Most respondents required a degree of time to understand the implications of the treatment and this led them to change their focus of attention as they considered the question more deeply. The specific codes went some way to help identify the different stages in the decision process but in some cases it 
may have been difficult to identify the respondent's overriding concerns. The analysis produced 14 general codes and 133 specific codes.

\section{Results}

\section{Quantitative results}

The first test of independence compared differences between health profiles and considered two cases. In one case, health states were compared in periods two and three (table 3 ). In this case respondents viewed the differences between $\mathrm{NN}$ and $\mathrm{YZ}$ differently depending upon the profile from which they were drawn, and these differences were statistically significant (two tailed $\mathrm{P}=$ 0.0048 based on the Wilcoxon matched-pairs test denoted by Wilcoxon Z test in tables 3 and 4$)^{6}$. Most respondents (21/29 respondents) gave ZNN - ZYZ > NNN - NYZ (question 5 - question $9>\mathrm{NNN}$ - question 7). In the other case, health states were compared in period three (table 4). However, the differences were not statistically different $(\mathrm{P}=0.0656)$ with only 18 of the 29 respondents viewing differences depending upon the profile from which they were drawn (i.e. giving ZYN-ZYZ > YYN - YYZ). It is possible that the inability to detect statistically differences in this latter case were due to the low power of the test. The independence tests were based on detecting a difference of 0.1 , with a standard deviation of 0.2 to ensure that the tests had an $80 \%$ chance of detecting statistical differences. The mean differences between the profiles in each case were 0.101 and 0.062 and the standard deviations were 0.164 and 0.152 respectively. This may explain why we were able to detect statistical differences for the former but not the latter case, since the power to detect differences in the first case was $91 \%$ and in the second was only $60 \%$.

Table $3 \mathrm{NN}$ - YZ in periods 2 and 3

Table $4 \mathrm{~N}-\mathrm{Z}$ in period 3

In the second test of independence, the SG1 question, respondents were asked whether they 
preferred a treatment that offered more intermittent states, or one that offered chronic states, or if they did not mind which treatment they received (i.e. they could express indifference between the two treatments). The sample was split into those preferring treatment $\mathrm{C}$ or treatment $\mathrm{D}$ (13 prefer C, 15 prefer D), with only one respondent expressing no preference between the two treatments.

In the dynamic consistency test, question SG3, it was assumed that YYZ was perceived as worse than YYN and better than YYD: responses were excluded if they perceived YYD to be better than YYZ (four respondents) or perceived YYN to be worse than YYZ (1 respondent). When these responses were excluded, the quantitative data showed that 17 respondents gave a higher probability of success in SG3 than that implied by questions 10, 4 and 6 (the implied SG3 probability, 17 respondents); however, the difference was not statistically significant ( $\mathrm{P}=$ 0.0520). The mean difference for this question was 0.159 and the standard deviation was 0.355 and the power of the test was $63 \%$.

The consistency check, question 11, assumed that the utility value for question 5 was higher than the utility value for question 2: three respondents were excluded from the analysis since they failed to comply with this requirement. The mean and median value of $\mathrm{Y}$ based on question 11 were 0.761 and 0.764 respectively, and 0.777 and 0.800 for $\mathrm{Y}$ based on question 2 (table 1). The value for $\mathrm{Y}$ in question 11 was not statistically different from the value for $\mathrm{Y}$ in question 2 ( $\mathrm{P}=$ $0.7879)$.

Richardson et al. (1989) found that constituent health states overestimated declining profiles whilst Dolan \& Gudex (1995) found they overestimated temporarily-deteriorating profiles. To compare the findings here with these we compared values based on constituent states to values based on profiles for a $0,5 \%$ and $10 \%$ discount rate. In questions 5, 6, 8 and 9 , the profile values were best estimated by imposing a zero discount rate on the constituent states (table 5). However, in the questions that involved a decline in health (YYZ, NYZ and YYD), the profile 
values were best estimated by imposing a positive discount rate on the constituent states. For instance, NYZ seemed to be best estimated by a $10 \%$ discount rate. Interestingly, the need to impose both a zero and $10 \%$ rate to explain the patterns of answers supports the conclusion that the implied rate of time preference varies across questions.

Table 5 The median values for profiles estimated directly and by their constituent states and at $0,5 \%$ and $10 \%$ discount rate

The Wilcoxon matched-pairs test was used to check whether the differences were statistically significant for a zero discount. These showed that the profile values for questions 5 and 9 were statistically different from the values based on the constituent states at a 0.05 significance level $(\mathrm{P}=0.0049$ and $\mathrm{P}=0.0094$ respectively). In profile $\mathrm{ZYZ}$ the constituent states overestimated the profile whilst in profile $\mathrm{ZNN}$ the constituent states underestimated the profile.

\section{Qualitative results}

This section considers the extent to which the qualitative data add to our understanding of the quantitative results in questions SG1-SG3.

In question SG1, the sample was split almost equally between respondents who preferred the intermittent treatment $\mathrm{C}$ and those who preferred the chronic treatment $\mathrm{D}$. The main themes (general codes) arising in the qualitative data are summarised in table 6. For instance, 7 of the 13 respondents preferring treatment $\mathrm{C}$ mentioned that treatment $\mathrm{C}$ offered a mixture of time in $\mathrm{N}$ and $\mathrm{Z}$ (row 1, table 6). For example,

Respondent: Go for treatment C, because either way I do not lose out since I get 3 plus 7 of full health whereas treatment $D$ is all or nothing. I am a 'settle for what I have' kind of a person. 
In addition, 5 of the 13 respondents preferring treatment $C$ mentioned that treatment $C$ was better if it failed (row 2, table 6). For example,

Respondent: I would not really want treatment $C$. I think that the option of $3 \operatorname{good}(N)$ and $7(Z)$ bad does not appeal to me at all. But looking at it again treatment $C$ may be better, whereas treatment $D$ is all or nothing..I think I would prefer treatment $C$ to have some time clear of pain and discomfort. I wouldn't like to think $50 \%$ if it went the wrong way in treatment D. In treatment $C$, if it went wrong at least you would get some pain-free days.

Turning to treatment $\mathrm{D}, 9$ of the 15 respondents preferring treatment $\mathrm{D}$ mentioned that it involved a cure or complete alleviation of the symptoms (row 4, table 6). For example,

Respondent: Treatment $D$ is the best since 50\% chance of total alleviation of all problems $(N)$. On the negative side treatment $C$ if it fails it is a better alternative as getting 3 years of remission..Sometimes it is easier when the option is death as you don't have to suffer. I would be less willing to go for treatment D since it is pink (Z) rather than death if it fails. But still go for treatment $D$ at 50:50.

In addition, 4 respondents preferring treatment $\mathrm{D}$ mentioned that treatment $\mathrm{C}$ still involved time in $\mathrm{Z}$ (row 5, table 6). One respondent preferred treatment $\mathrm{D}$ since they felt that treatment $\mathrm{C}$ failed to give a clear outcome (row 6, table 6). Three respondents felt that the treatments were similar (rows 3,7 , and 8 , table 6), but only one of these expressed indifference (row 7, table 6).

In question SG3, postponing the risky treatment until the beginning of the seventh year was not statistically significant. In addition, the responses were qualitatively different to our predictions. We had expected that the probability of success for question SG3 would have been lower than that implied by questions 10, 4 and 6 (the implied SG3 probability). In particular, we had anticipated that respondents would accept a higher probability of failure since health deteriorates from $\mathrm{Y}$ to $\mathrm{Z}$ in the seventh year. Though not statistically significant the results showed that 17 of the 24 respondents gave a higher probability of success for SG3 than that implied for SG3. 
The qualitative data in table 7 offer an explanation for this and support the notion that half the respondents who mentioned the worsening profile failed to adjust their responses sufficiently and gave a higher probability of success for SG3 (row 1, table 7). The following is an illustration of this type of response:

Respondent: SG3 I think go down more in terms of 60\%. This way round (green (Y) then pink $(Z)$, rather than pink (Z) then green (Y) as in SG2), if there is the possibility of improving in the last 4 years (to N) more like $60 \%$. I think the difficult thing has gone really. I would not want to go through treatment $H$ (with pink $Z$ at end). If it came to the crunch I would take treatment $G$ if very low.

Table 7 A comparison of SG3 probabilities

A further two respondents (row 4, table 7) simply stated that they were willing to accept a lower probability of success but did not give a clear reason for this answer. They were again split between giving a higher or lower probability of success for SG3 compared to that implied for SG3, and it was suspected that they were again thinking in terms of the worsening scenario.

Six respondents mentioned the importance of the last 4 years in normal health (row 2, table 7). For example,

Respondent: SG3. Prefer treatment $G$, as in treatment $H$ seem in discomfort in pink (Z) before you die,...so would take a low chance of success and still prefer treatment G,..I would not want my family to see me in Z..prefer to go for treatment $G$ and hope it works at the end and get to that (N).

Again, 4 of these 6 respondents gave a higher probability of success for SG3 than that implied for SG3. One respondent did not want to undergo $\mathrm{Z}$ and gave a lower probability of success for SG3 than that implied for SG3 (row 6, table 7), supporting the notion that they would take more risk if they were unwilling to undergo $\mathrm{Z}$. 
There was evidence, however, that other factors were at work which increased the SG3 probability relative to the implied SG3 probability. For instance, there was an indication that respondents' attitude to the risk of death may have changed. When considering only 4 years, respondents may have become more risk-averse and demanded a higher probability of success in SG3. Qualitative evidence that could be cited as evidence of this centres around six respondents who mentioned that it was not worth dying to get the last 4 years in normal health (row 3, table 7). For example,

Respondent: It is the black oblong (death, D). Pink (Z) is still better then black (D). You haven't really suffered for the first 6 years (in green Y), so you are looking forward to the last 4 years taking away some anxiety and depression. If you did have 4 years in white (N) you could still die, and in pink (Z) at least you are still alive. If being poorly for 6 years,..well by then you are used to doctors and nurses caring for you and not so worried about washing and dressing and walking about you will become part of the big wheel. In the first 6 years relatively capable (in Y) and 4 years stuck in pink (Z).

Other responses in this category related to the tolerance of the condition and family/child care commitments. The quantitative data showed that these respondents were less willing to accept the risk of failure, with all respondents in this category giving a higher probability of success for SG3 than that implied for SG3. In addition, two respondents linked their responses back to 10 years in $Z$ (row 5, table 7) feeling that they required a higher probability of success in the SG3 question: one of these felt that it should be higher, since the treatment offered only 4 years of normal health, whilst the other felt that 4 years in $\mathrm{Z}$ was more bearable. Their quantitative responses confirmed their verbal accounts and both gave a higher probability of success for SG3 compared to the implied SG3 probability. In addition, both of these respondents demanded a higher probability of success than they had under 10 years in Z (the SG3 probability is 0.980 and 0.9 compared to probability of success of 0.575 and 0.5 respectively for $Z$ ). In summary, the analysis suggested that some respondents are more risk-averse in the last four years (two respondents state this explicitly) and all of these gave a higher probability of success for SG3 compared to that implied for SG3. 


\section{Concluding remarks}

The main test considered here, the test of differences, bypasses the problems of the changing attitudes towards time preference encountered in tests that rely on estimating profiles from constituent states. We are unable to conclusively reject additive independence in this test, with only one of the two tests detecting statistical differences. In the other test of independence, the sample was split almost equally between respondents that preferred the intermittent treatment $\mathrm{C}$ and those that preferred the chronic treatment D. Finally, respondents' preferences are found to be consistent over time.

It can be argued that the study was too complex and that respondents had difficulty estimating mixed profiles. However, this appears not to have been a problem as the standard deviations of questions 1 to 10 are similar to those reported by other studies, and we are unable to refute the test of consistency (question 11).

How useful was the qualitative approach? The qualitative data helped to reveal underlying themes arising in the responses. In the choice between treatments $\mathrm{C}$ and $\mathrm{D}$, without the qualitative data, we might conclude that respondents chose randomly between the treatments even though they were given the option of saying that they were indifferent between the two treatments. The qualitative data suggest, instead, that the sample had contrasting viewpoints about the benefits derived from such choices. In the test that compared preferences over time, the qualitative data offer an explanation for the failure to find differences. In particular, half of the respondents who mentioned the worsening scenario failed to adjust their responses. This is in keeping with the conclusion of Dolan \& Gudex (1995) that respondents fail to adequately adjust responses. There is evidence also that other factors were at work which increased the SG3 probability relative to the implied SG3 probability. For instance, there was an indication that respondents' attitude to the risk of death may have changed.

The paper also suggests the limitations of the qualitative data and the analysis that was performed. In an open-ended questionnaire, a respondent may not always address the issue that 
the experimenter is most interested in, and the experimenter feels inhibited to ask the question just in case it biases the answers. One possibility for future work is for a more structured approach to gathering qualitative data, which enables the researcher to develop closer links to the quantitative data. For example, respondents could be asked to consider a list of factors and to confirm which were important to their decision. Such an approach would standardise the procedure and reduce bias by encouraging respondents to think of a variety of factors. It is also apparent that the data analysis developed a microcosm of interpretations (specific codes) and increased the number of subdivisions that could be made in the quantitative data. These subdivisions sometimes made it hard to make meaningful comparisons between the qualitative and quantitative data as there were few observations falling into each theme. The analysis was more successful in explaining why the quantitative results failed to be statistically different, showing the counteracting influences that were affected the results.

Which profiles are prone to failures of additive independence? For this we return to the profiles that formed part of the main independence test that was found to be statistically different. In this case we found that respondents viewed the differences between $\mathrm{NN}$ and YZ differently depending upon the profile which they were drawn: and that differences in values between profiles $\mathrm{ZNN}$ and $\mathrm{ZYZ}$ were greater than the differences in values between profiles NNN and NYZ. This could arise from one or more of the following: profile ZNN was valued higher or profiles ZYZ and NYZ were valued lower than additive independence would predict. An indication of the profiles that are driving this result can be found by looking again at the estimation of profiles from constituent states. In the case of a zero discount rate considered by Dolan \& Gudex (1995), we found that constituent states overestimated the temporarilyimproving profile $\mathrm{ZYZ}$ and constituent states underestimated the improving profile ZNN. From this we tentatively conclude that profiles $\mathrm{ZYZ}$ and $\mathrm{ZNN}$ led to a failure of additive independence in our study.

The extent to which sequencing issues arise appears to be heavily dependent upon the profile and the viewpoint about the benefit derived from such profiles. This element of subjectivity is in 
keeping with the notion respondents preferences for health treatments are related to their expectations of health (Chapman, 1996). Patterns are beginning to emerge in the empirical work about the instances in which additive independence does not hold, but our tests are unable to conclusively reject additive independence. At the moment the best way forward would be to include estimation of profiles and constituent states to check the extent to additive independence continues to hold. 


\section{References}

Beattie, J., Covey, J., Dolan, P., Hopkins, L., Jones-Lee, M., Loomes, G., Pidgeon, N., Robinson, A., \& Spencer, A. (1998). On the contingent valuation and the safety of contingent valuation: part 1, Journal of Risk and Uncertainty 17, 5-25.

Bleichrodt, H. (1995). QALYS and HYEs: Under what conditions are they equivalent? Journal of Health Economics 14, 17-37.

Chapman, G.B. (1996). Expectations and preferences for sequences of health and money. Organizational Behavior and Human Decision Processes 67, 59-75.

Charmaz, C. (1990). Discovering chronic illness: using grounded theory, Social Science and Medicine 30, 1161-1172.

Curtis, S., Gesler, W., Smith, G., \& Washurn, S. (2000). Approaches to sampling and case selection in qualitative research: examples in the geography of health, Social Science and Medicine 50, 1001-1014.

Dolan, P., \& Cookson, R. (2000). A qualitative study of the extent to which health gain matters when choosing between groups of patients, Health Policy 51, 19-30.

Dolan, P., Cookson, R., \& Ferguson, B. (1999). Effect of discussion and deliberation on public's views about priority setting in health care: focus group study. British Medical Journal 318, 916-919.

Dolan, P., \& Gudex C. (1995). Time preference, duration and health state valuations. Health Economics 4, 289-299.

Drummond, M.F., O'Brien, B., Stoddart, G.L., \& Torrance, G.W. (1997). Methods for Economic Evaluation of Health Care Programmes. Oxford Medical Publications, Second Edition.

Ericsson, K.A., \& Simon, H.A. (1980). Verbal reports as data, Psychological Review 87, 215-251.

Gafni, A. (1995). Time in health: can we measure individuals" "pure time preference". Medical Decision Making 15, 31-37.

Glaser, B.G., \& Strauss, A.L. (1967). The discovery of grounded theory: strategies for qualitative research, first edition, Aldine de Gruyter, New York. 
Holmes, A.M. (1998). Measurement of short term health effects in economic evaluations. Pharmacoeconomics 13, 171-174.

Johnston, K., Brown, J., Gerard, K., \& O’Hanlon \& Morton, A. (1998), Valuing temporary and chronic health states associated with breast screening. Social Science and Medicine 47, 213-222.

Jones-Lee, M.W., Loomes, G., \& Philips, P. (1995). Valuing the prevention of non-fatal road injuries: contingent valuation versus standard gamble. Oxford Economic Papers 47, 676695.

Kahneman, D., Fredrickson, B.L., Schreibner, C.A., \& Redelmeier, D.A. (1993). When more pain is preferred to less: adding a better end. Psychological Science 4, 401-405.

Kidder, L.H. (1981). Qualitative research and quasi-experimental frameworks, In Scientific Inquiry and the social sciences, Brewer, M.B. and Collins, B.E. (eds.), Jossey-Bass, San Francisco, 226-256.

Kind, P., Dolan, P., Gudex, C. \& Williams, A. (1998). Variations in population health status: results from a United Kingdom national questionnaire survey. British Medical Journal 16, 736-741.

Krabbe, P.F., \& Bonsel, G.J. (1998). Sequence effects, health profiles, and the QALY model: in search of realistic modelling. Medical Decision Making 18, 178-186.

Kuppermann, M., Shiboski, S., Feeny, D., Elkin, E.P. \& Washington, A.E. (1997) Can preference scores for discrete states be used to derive preference scores for an entire path of events? Medical Decision Making 17, 42-55.

Lipscomb, J. (1989). The preference for health in cost-effectiveness analysis. Medical Care 27, S233-53

Loewenstein, G., \& Prelec, D. (1993). Preferences for sequences of outcomes. Psychological Review 100, 91-108.

Loomes, G., \& McKenzie, L. (1989). The use of QALYs in health care decision making. Social Science and Medicine 28, 299-308. 
Mackeigan, L.D., O'Brien, B.J., \& Oh, P.I. (1999). Holistic versus composite preferences for lifetime treatment sequences for type 2 diabetes. Medical Decision Making 19, 113-121. Patton, M.Q. (1990). Qualitative Evaluation and Research Methods. London: Sage Publications. Pliskin, J.S., Shepard, D.S., \& Weinstein, M.C. (1980). Utility functions of life-years and health status. Operational Research 28, 206-224.

Richardson, J., Hall, J., \& Salkeld, G. (1996). The measurement of utility in multiphase health states. International Journal of Technology Assessment in Health Care 12, 151-162.

Richardson, J., Hall, J., \& Salkeld, G. (1989). The compatibility of measurement techniques and the measurement of utility through time, In Economics and Health: proceedings of the eleventh conference of health Economics, Smith, C.S. (ed), Melborne: Public Sector management institute, Monash university, 1989.

Ross, W.T., \& Simonson, I. (1991). Evaluating pairs of experiences: a preference for happy endings. Journal of Behavioral Decision Making 4, 273-282.

Robinson, A., Dolan, P., \& Williams, A. (1997). Valuing health status using VAS and TTO: What lies behind the numbers? Social Science and Medicine 45, 1289-1297.

Spencer, A. (1999). The independence axiom and health state valuation. Studies in health economics 27, Lund University, Department of Community Medicine, Malmö, Sweden.

Spencer, A. (1998). Value elicitation techniques: methodological issues involved in two-stage procedures. D.Phil, University of York.

Torrance, G.W. (1986). Measurement of health state utilities for economic appraisal. Journal of Health Economics 5, 1-30.

Treadwell, J.R. (1998). Tests of preferential independence in the QALY model. Medical Decision Making 18, 418-428.

Varey, C., \& Kahneman, D. (1992). Experiences extended across time: evaluation of moments and episodes. Journal of Behavioral Decision Making 5, 169-185.

von Winterfeldt, D., \& Edwards, W. (1986). Decision analysis and behavioral research. first edition, Cambridge University Press, Cambridge Mass. 
Testing the additive independence assumption of the QALY model

Table 1

An overview of the questionnaire

\begin{tabular}{|c|c|c|c|c|}
\hline (1) & $\begin{array}{c}(2) \\
\text { Profiles }\end{array}$ & $\begin{array}{c}(3) \\
\text { mean }\end{array}$ & $\begin{array}{c}(4) \\
\text { media } \\
n\end{array}$ & $\begin{array}{c}\text { (5) } \\
\text { standard } \\
\text { deviation }\end{array}$ \\
\hline 1 & WWW & 0.800 & 0.825 & 0.170 \\
\hline 2 & YYY & 0.777 & 0.800 & 0.142 \\
\hline 3 & $\mathrm{ZZZ}$ & 0.461 & 0.450 & 0.245 \\
\hline 4 & YYZ & 0.642 & 0.660 & 0.184 \\
\hline 5 & $\mathrm{ZNN}$ & 0.903 & 0.950 & 0.110 \\
\hline 6 & YYN & 0.875 & 0.925 & 0.117 \\
\hline 7 & NYZ & 0.707 & 0.750 & 0.198 \\
\hline 8 & $\mathrm{ZYN}$ & 0.803 & 0.850 & 0.149 \\
\hline 9 & ZYZ & 0.508 & 0.500 & 0.230 \\
\hline 10 & YYD & 0.482 & 0.500 & 0.236 \\
\hline Q11 & Consistency check & 0.761 & 0.764 & 0.155 \\
\hline SG1 & $\begin{array}{l}\text { Chronic and intermittent } \\
\text { treatment }\end{array}$ & $\mathrm{n} / \mathrm{a}$ & $\mathrm{n} / \mathrm{a}$ & $\mathrm{n} / \mathrm{a}$ \\
\hline SG2 & Postponing treatment & 0.765 & 0.800 & 0.192 \\
\hline SG3 & Postponing treatment & 0.592 & 0.650 & 0.279 \\
\hline
\end{tabular}


Table 2

The sample

\begin{tabular}{|l|c|c|c|}
\hline \multicolumn{1}{|c|}{$(1)$} & $\begin{array}{c}(2) \\
18-39\end{array}$ & $\begin{array}{c}(3) \\
40-59\end{array}$ & $\begin{array}{c}(4) \\
\text { Total }\end{array}$ \\
\hline Male & $7(\%)$ & $8(\%)$ & $15(\%)$ \\
\hline Female & $10(\%)$ & $4(\%)$ & $14(\%)$ \\
\hline Total & 17 & 12 & 29 \\
\hline
\end{tabular}


Table 3

$\mathrm{NN}-\mathrm{YZ}$ in periods 2 and 3

\begin{tabular}{|c|c|}
\hline \multicolumn{2}{|r|}{ Qi compared to Qj } \\
\hline \multirow[t]{4}{*}{ (1) } & (2) \\
\hline & NN-YZ periods 2 and 3 \\
\hline & Q5-Q9 compared NNN-Q7 \\
\hline & i.e. ZNN - ZYZ compared NNN - NYZ \\
\hline (1) $Q i>Q j$ & 21 \\
\hline (2) $\mathrm{Qi}<\mathrm{Qj}$ & 5 \\
\hline (3) $\mathrm{Qi}=\mathrm{Qj}$ & 3 \\
\hline (4) Total & 29 \\
\hline (5) Wilcoxon Z & -2.8192 \\
\hline (6) 2-tailed P & 0.0048 \\
\hline
\end{tabular}


Table 4

$\mathrm{N}-\mathrm{Z}$ in period 3

\begin{tabular}{|c|c|}
\hline \multicolumn{2}{|r|}{ Qi compared Qj } \\
\hline \multirow[t]{4}{*}{ (1) } & $(2)$ \\
\hline & $\mathrm{N}-\mathrm{Z}$ period 3 \\
\hline & Q8-Q9 compared Q6-Q4 \\
\hline & i.e. ZYN - ZYZ compared YYN - YYZ \\
\hline (1) Qi $>$ Qj & 18 \\
\hline (2) $\mathrm{Qi}<\mathrm{Qj}$ & 8 \\
\hline (3) $\mathrm{Qi}=\mathrm{Qj}$ & 3 \\
\hline (4) Total & 29 \\
\hline (5) Wilcoxon Z & -1.8414 \\
\hline (6) 2-tailed P & 0.0656 \\
\hline
\end{tabular}


Table 5

The median values for profiles estimated directly and by their constituent states and a $0,5 \%$ and $10 \%$ discount rate

\begin{tabular}{|c|c|c|c|c|}
\hline$(1)$ & $\begin{array}{c}(2) \\
\text { direct value }\end{array}$ & $\begin{array}{c}(3) \\
0 \%\end{array}$ & $\begin{array}{c}(4) \\
5 \%\end{array}$ & $\begin{array}{c}(5) \\
10 \%\end{array}$ \\
\hline 4 & 0.660 & 0.630 & 0.647 & 0.674 \\
\hline 5 & 0.950 & 0.835 & 0.806 & 0.777 \\
\hline 6 & 0.925 & 0.880 & 0.869 & 0.858 \\
\hline 7 & 0.750 & 0.680 & 0.713 & 0.751 \\
\hline 8 & 0.850 & 0.745 & 0.705 & 0.676 \\
\hline 9 & 0.500 & 0.530 & 0.530 & 0.530 \\
\hline 10 & 0.500 & 0.480 & 0.526 & 0.567 \\
\hline
\end{tabular}


Table 6

Factors considered in the SG1 question

\begin{tabular}{|c|c|c|}
\hline & $\begin{array}{l}\text { (1) } \\
\text { rationale for choice }\end{array}$ & $\begin{array}{c}\text { (2) } \\
\text { number of respondents }\end{array}$ \\
\hline \multirow[t]{3}{*}{ Treatment $\mathrm{C}$} & $\begin{array}{l}\text { (1) treatment } \mathrm{C} \text { offers a mixture of } \\
\text { time in } \mathrm{N} \text { and } \mathrm{Z}\end{array}$ & $\begin{array}{c}7 \\
\text { (resp. 4, 6, 7, 11, 16, } 19 \text { and } \\
\text { 23) }\end{array}$ \\
\hline & $\begin{array}{l}\text { (2) respondents were concerned } \\
\text { about the failure outcome }\end{array}$ & $\begin{array}{c}5 \\
\text { (resp. 5, 20, 24, 27 and 28) }\end{array}$ \\
\hline & (3) treatment C and D seem similar & $\begin{array}{c}1 \\
\text { (resp. 17) }\end{array}$ \\
\hline \multirow[t]{4}{*}{ Treatment D } & (4) treatment D offers a cure & $\begin{array}{c}9 \\
(\text { resp. 1, 8, 9, 13, 18, 21, 22, } \\
\text { 25 and 29) }\end{array}$ \\
\hline & $\begin{array}{l}\text { (5) treatment } \mathrm{C} \text { still leads to a } \\
\text { substantial time in } \mathrm{Z} \text { or still have to } \\
\text { undergo } \mathrm{Z}\end{array}$ & $\begin{array}{c}4 \\
\text { (resp. 2, 3, } 10 \text { and 12) }\end{array}$ \\
\hline & (6) treatment $\mathrm{C}$ is wishy washy & $\begin{array}{c}1 \\
(\text { resp. 26) }\end{array}$ \\
\hline & (7) treatment $\mathrm{C}$ and $\mathrm{D}$ seem similar & $\begin{array}{c}1 \\
\text { (resp. 15) }\end{array}$ \\
\hline No difference & (8) treatment C and D seem similar & $\begin{array}{c}1 \\
\text { (resp. 14) }\end{array}$ \\
\hline
\end{tabular}

Parentheses indicate the respondents who fall under each category (by respondent number) and the abbreviation resp. denotes respondent. 
Table 7 A comparison of SG3 probabilities

\begin{tabular}{|c|c|c|c|}
\hline \multicolumn{4}{|c|}{ A comparison of the probabilities of success (by number of respondents) } \\
\hline (1) & $\begin{array}{l}(2) \\
\text { implied SG3 > } \\
\text { SG3 }\end{array}$ & $\begin{array}{c}(3) \\
\text { implied SG3 }<\text { SG3 }\end{array}$ & $\begin{array}{c}(4) \\
(n=29)\end{array}$ \\
\hline $\begin{array}{l}\text { (1) worsening scenario, } \mathrm{n}=12 \\
\text { (resp. } 2,6,8, \mathbf{1 1}, 15,16, \mathbf{1 7}, 18 \text {, } \\
22,24,25 \text { and } 29 \text { ) }\end{array}$ & $\begin{array}{c}6 \\
\text { (resp. } 2, \mathbf{1 1}, 15 \text {, } \\
18,24 \text { and } 29)\end{array}$ & $\begin{array}{l}6 \\
\text { (resp. } 6,8,16,17 \\
22 \text { and 25) }\end{array}$ & 12 \\
\hline $\begin{array}{l}\text { (2) last } 4 \text { years important to have } \\
\text { good health, } \mathrm{n}=6 \\
\text { (resp. } 1, \mathbf{5}, \mathbf{7}, 10, \mathbf{1 2} \text { and } 21 \text { ) }\end{array}$ & (resp. 7, and 12) & $\begin{array}{c}4 \\
\text { (resp. 1, 5, } 10 \text { and } \\
21 \text { ) }\end{array}$ & 6 \\
\hline $\begin{array}{l}\text { (3) better to live than die and } \\
\text { want a high success, } n=6 \\
\text { (resp. } 3,9,13,14,23 \text { and } 26 \text { ) }\end{array}$ & 0 & $\begin{array}{c}6 \\
\text { (resp. } 3,9,13,14 \\
23 \text { and 26) }\end{array}$ & 6 \\
\hline $\begin{array}{l}\text { (4) Low success, } n=2 \\
\text { (resp. } 4,19)\end{array}$ & $\begin{array}{c}1 \\
\text { (resp. 4) }\end{array}$ & $\begin{array}{c}1 \\
(\text { resp. 19) }\end{array}$ & 2 \\
\hline $\begin{array}{l}\text { (5) If } 10 \mathrm{Z} \text { lower success, } \mathrm{n}=2 \\
\text { (resp. 20, 27) }\end{array}$ & 0 & $\begin{array}{c}2 \\
(\operatorname{resp} 20,27)\end{array}$ & 2 \\
\hline $\begin{array}{l}\text { (6) Not want } Z, n=1 \\
\text { (resp. 28) }\end{array}$ & $\begin{array}{c}1 \\
\text { (resp. 28) }\end{array}$ & 0 & 1 \\
\hline
\end{tabular}

Bold indicates those responses that were eliminated. See the quantitative results section for an explanation of why there respondents were excluded.

Parentheses indicate the respondents who fall under each category (by respondent number) and the abbreviation resp. denotes respondent. 
Figure 1

Four health profiles
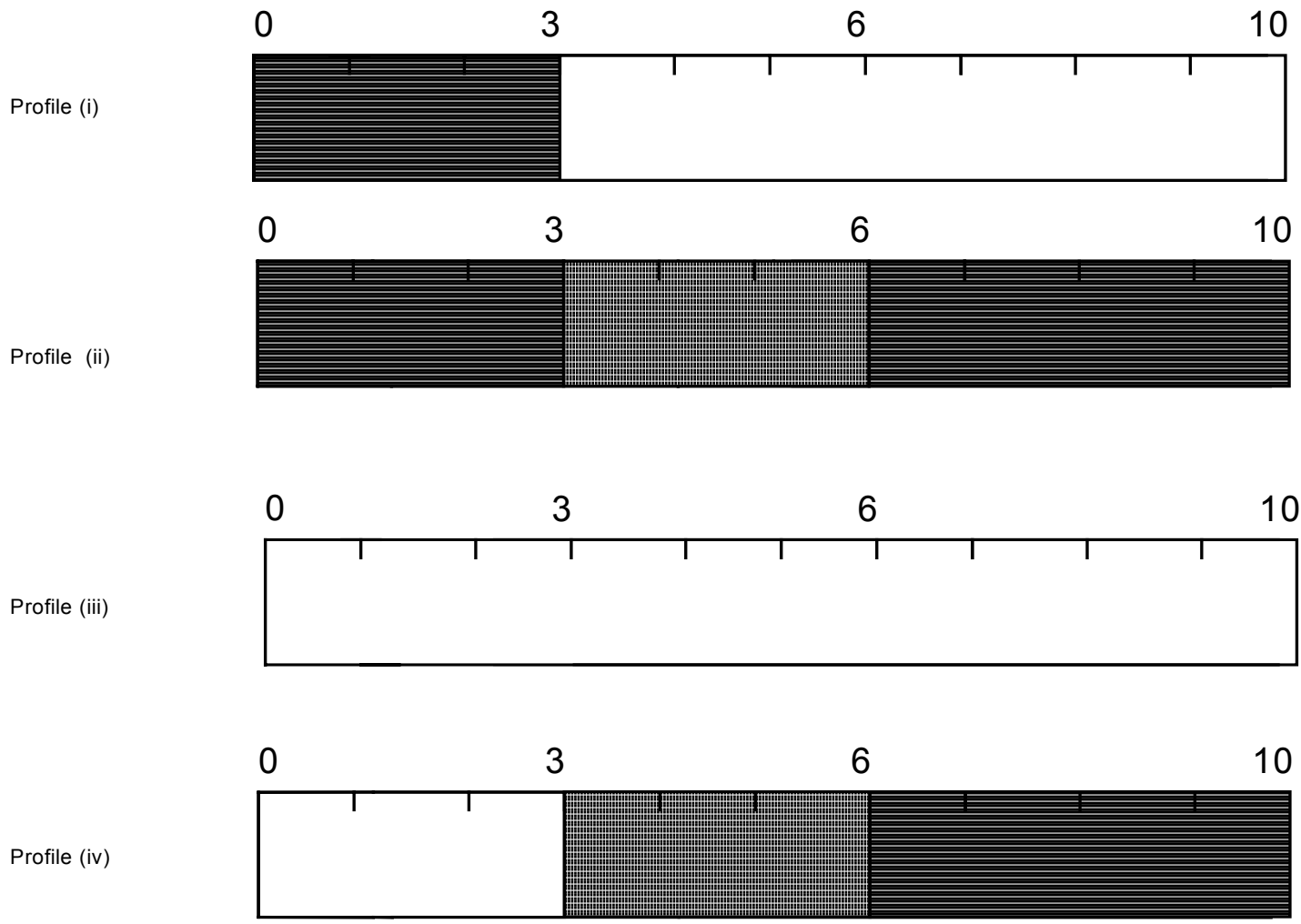
Figure 2

Chronic and intermittent health states

Treatment $\mathrm{C}$

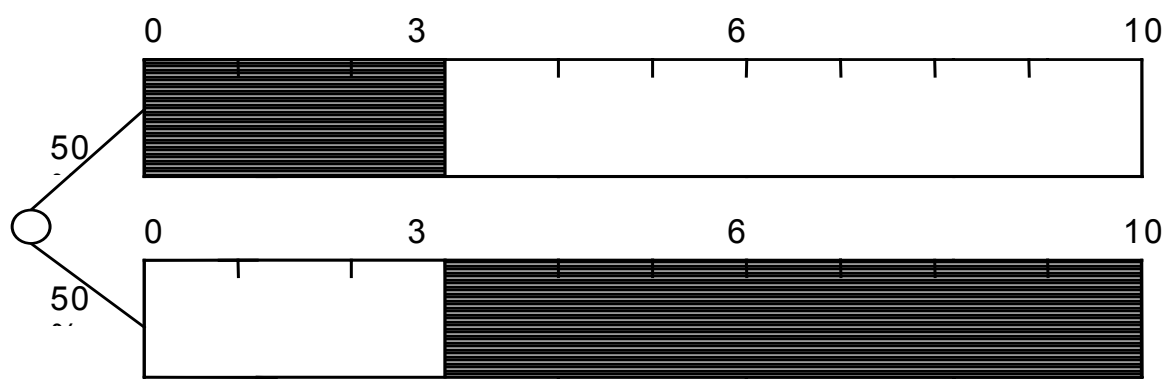

Treatment D

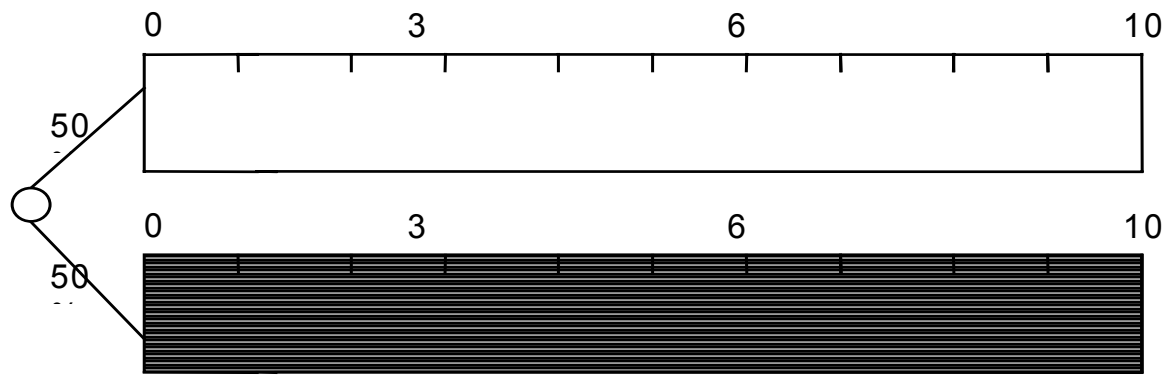


Figure 3

Postponing treatment in question SG3

Treatment G

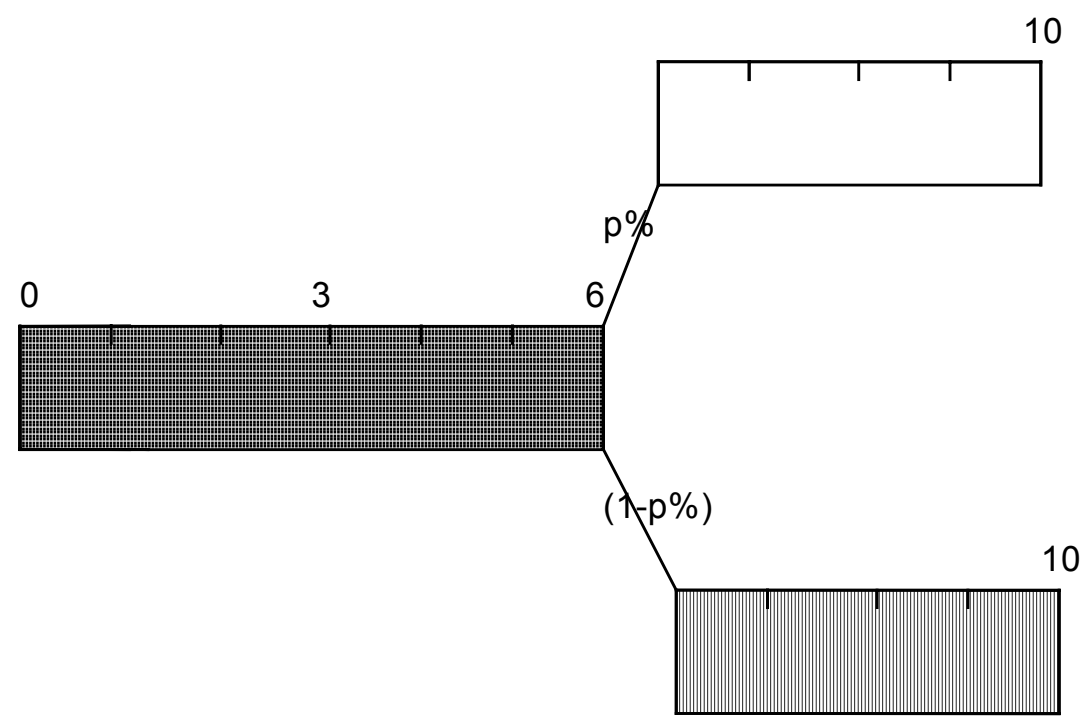

Treatment $\mathrm{H}$

36

10

$100 \%$

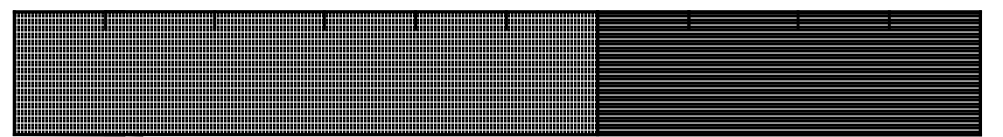


Figure 4

The implied SG3 question

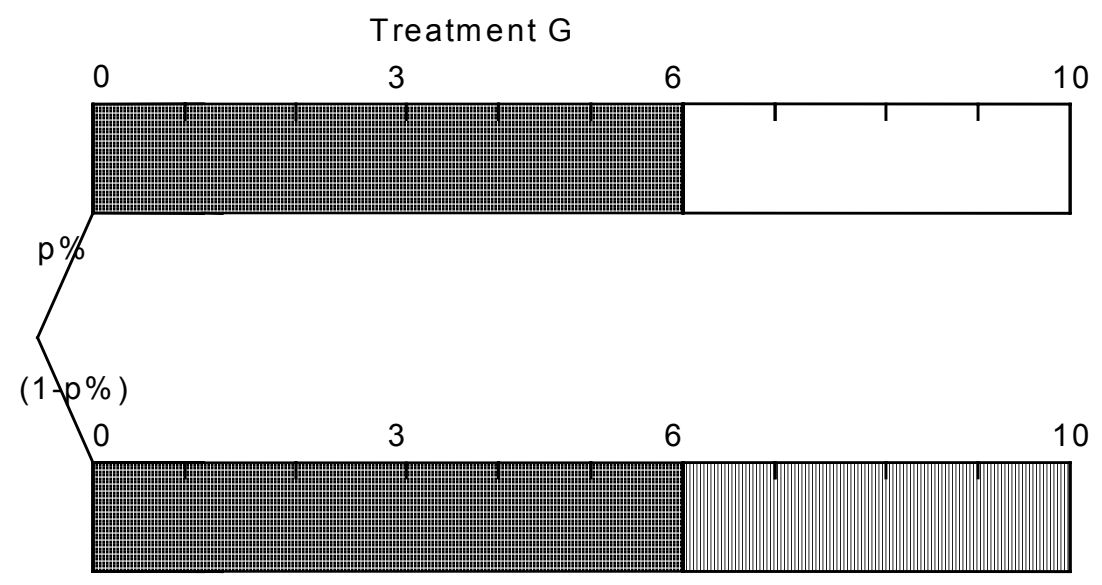

$100 \%$

Treatment $\mathrm{H}$

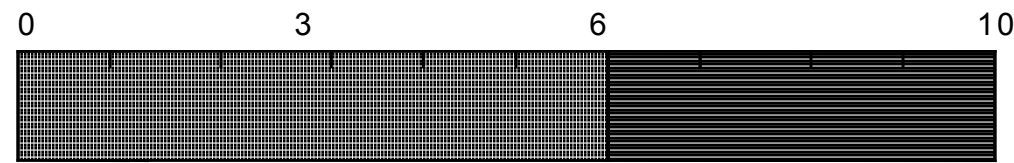


Figure 5

The format of questions 1 to 10

Treatment A

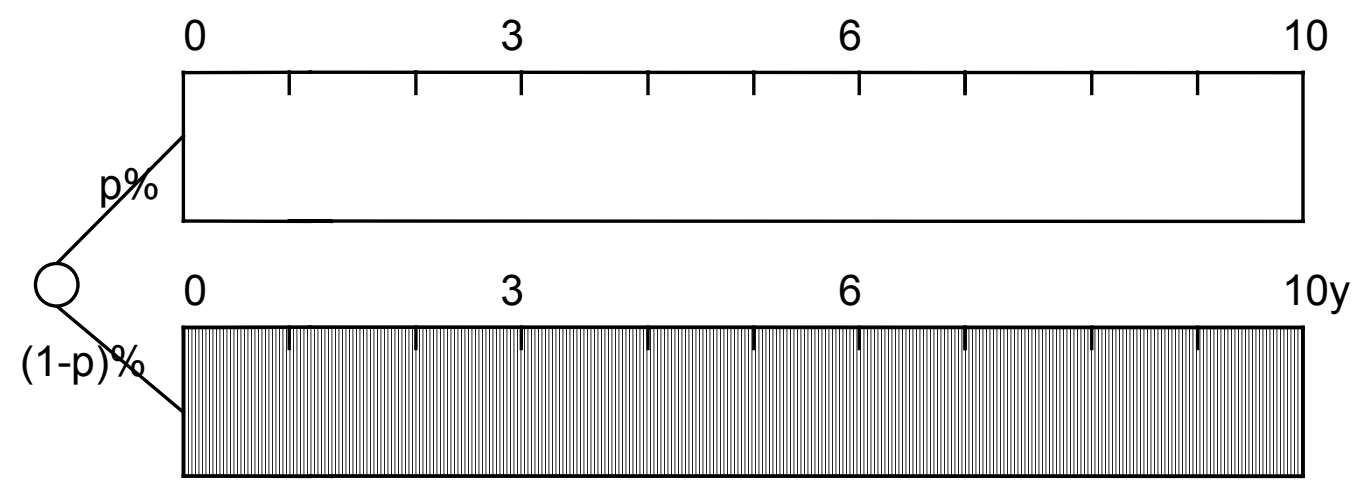

Treatment B

Place each card in turn 


\section{Acknowledgements}

The author would like to thank Graham Loomes, Karl Claxton, Judith Covey, Angela Robinson, Paul Dolan, Nick Pidgeon and Carl Hampus Lyttkens for their valuable comments. In addition, the author is grateful for Lund University for funding a research fellowship to pilot the approach and ESRC money to finance a UK study. Any errors are the responsibility of the author alone.

\footnotetext{
${ }^{1}$ In the ranking and valuation questions women were asked to imagine that each state lasted for the remainder of their expected life span, rounded to the nearest 10 years.

${ }^{2}$ The last three are based on three cancer states, although the respondents were unaware of this.

${ }^{3}$ More complex models, for instance the multiplicative (Bleichrodt, 1995) and multilinear models (von Winterfeldt \& Edwards, 1986), would have attempted to estimate the interrelationships between states.

${ }^{4}$ The predictive error indicates a better model. The general additive model had a predictive error of 0.19 compared to 0.35 for the additive model. At an individual level, the general additive model gave statistically significant coefficients on all time periods, except the first trimester.

${ }^{5}$ Respondents were recruited by a freelance market researcher.

${ }^{6}$ A two-tailed Kolmogorov-Smirnov test at a 0.05 significance level was used to test the hypothesis that data were normally distributed. The test statistic was sufficiently large to reject the null hypothesis. We, therefore, apply non-parametric tests in what follows.
} 


\section{Appendix 1}

Let $\boldsymbol{q}$ denote a vector of health states, $\boldsymbol{q}=\left(q_{1}, \ldots . . q_{n}\right)$, which the individual derives utility, $u_{t}(\boldsymbol{q})$. Suppose that the vector of attributes $\boldsymbol{q}$ can be subdivided into two subvectors $\boldsymbol{r}$ and $\boldsymbol{s}$ where

$\boldsymbol{r}=\left(r_{1} \ldots . . r_{i}\right)$ and $\boldsymbol{s}=\left(s_{i+1} \ldots s_{n}\right)$

Consider one value for the vector, $\boldsymbol{r}, \boldsymbol{r}^{l}=\left(r^{1}{ }_{1} \ldots . . r_{i}{ }_{i}\right)$ and two values for the vector $\boldsymbol{s}$, $\mathbf{s}^{1}=\left(\mathrm{s}_{\mathrm{i}+1}^{1} \ldots . \mathrm{s}_{\mathrm{n}}{ }_{\mathrm{n}}\right)$ and $\boldsymbol{s}^{2}=\left(s^{2}{ }_{i+1} \ldots . s_{n}{ }_{n}\right)$ then by definition of the independence axiom of expected utility:

$\left[\left(\boldsymbol{r}^{1}, \boldsymbol{s}^{1}\right) \succ\left(\boldsymbol{r}^{1}, \boldsymbol{s}^{2}\right)\right] \Rightarrow\left[\left(\boldsymbol{r}, \boldsymbol{s}^{1}\right) \succ\left(\boldsymbol{r}, \boldsymbol{s}^{2}\right)\right] \forall \boldsymbol{r}, \boldsymbol{r}^{1}, \boldsymbol{s}^{1}, \boldsymbol{s}^{2}$

In terms of utility functions the left-hand-side of this expression can be represented by:

$u_{t}\left(r^{1}, s^{1}\right) \geq u_{t}\left(r^{1}, s^{2}\right)$.

Under the additive independence assumption the utility function can be written as

$u_{t}(q)=\sum_{t=1}^{n} u_{t}\left(q_{t}\right)$, so the utility function can be written as:

$\sum_{t=1}^{n} u_{t}\left(r^{1}, s^{1}\right) \geq \sum_{t=1}^{n} u_{t}\left(r^{1}, s^{2}\right)$

This expands to:

$u_{1}\left(r_{1}^{l}\right)+. . u_{i}\left(r_{i}^{l}\right)+u_{i+1}\left(s_{i+1}^{l}\right)+. . u_{n}\left(s_{n}{ }_{n}\right) \geq u_{1}\left(r^{l}{ }_{1}\right)+. . u_{i}\left(r_{i}^{l}\right)+u_{i+1}\left(s^{2}{ }_{i+1}\right)+. . u_{n}\left(s^{2}{ }_{n}\right)$

$u_{i+1}\left(s_{i+1}^{1} s_{i+1}^{2}\right)+. . u_{n}\left(s_{n^{-}}^{1} s_{n}^{2}\right) \geq 0$

$\sum_{t=i+1}^{n} Q_{t}\left(s_{t+1}^{1}-s_{n}^{2}\right) \geq 0$

Therefore, the difference between the utilities in profiles reflects the utilities of the health states that are common to both profiles. 


\section{Q Queen Mary \\ University of London}

This working paper has been produced by the Department of Economics at Queen Mary, University of London

Copyright ( 2000 Anne Spencer All rights reserved.

\section{Department of Economics}

Queen Mary, University of London

Mile End Road

London E1 4NS

Tel: +44 (0)20 78825096 or Fax: +44 (0)20 89833580

Email: j.conner@qmw.ac.uk

Website: www.econ.qmw.ac.uk/papers/wp.htm 